\title{
SANTA CRUZ DE GUANACASTE: CULTURA LOCAL, TURISMO Y GLOBALIZACIÓN
}

\section{SANTA CRUZ, GUANACASTE: LOCAL CULTURE, TOURISM AND GLOBALIZATION}

\section{Luis Carlos Morales Zúñiga*}

\author{
Mi espíritu nunca muere porque ha nacido junto al corral, \\ babeado por los terneros y el calor de mi buena mama... \\ Medardo Guido Acevedo \\ Compositor y folclorista guanacasteco
}

\begin{abstract}
RESUMEN
El artículo analiza la relación entre la cultura local del cantón de Santa Cruz, Guanacaste, y el desarrollo de la actividad turística en este cantón. En un primer apartado se explora el origen y desarrollo de la cultura popular del cantón, vinculándola a las actividades tradicionales agropecuarias; seguidamente se describe el despliegue de la actividad turística en el cantón, con el fin de analizar la cultura popular de este cantón en un marco de reconversión productiva que va desde las actividades agropecuarias hacia el sector turístico, en un contexto de globalización económica y cultural.
\end{abstract}

PALABRAS CLAVE: COSTA RICA, GUANACASTE * SANTA CRUZ * CULTURA LOCAL * TURISMO * GLOBALIZACIÓN

\section{ABSTRACT}

The article analyzes the relationship between local culture of the canton of Santa Cruz, Guanacaste, and the development of tourism in the canton. In a first section explores the origin and development of popular culture in the canton, linking it to traditional farming activities, and then describes the deployment of tourism in Santa

Licenciado en la Enseñanza de los Estudios Sociales y la Educación Cívica. Egresado de la
Maestría Centroamericana en Sociología con sede en la Universidad de Costa Rica. luis23m@gmailcom 
Cruz, in order to analyze the popular culture of the canton in a framework of productive activities ranging from farming to tourism, in a context of economic and cultural globalization.

KEY WORDS: COSTA RICA, GUANACASTE * SANTA CRUZ * LOCAL CULTURE * TOURISM * GLOBALIZATION

\section{INTRODUCCIÓN}

Una de las particularidades destacables del cantón de Santa Cruz es su cultura local, construida y marcada por un proceso socio-histórico delineado por la preponderancia de las actividades agropecuarias. Tales actividades económicas han construido una cultura local donde muchas tradiciones están en función de la vida en torno a las haciendas ganaderas y sus actividades cotidianas, dando paso al surgimiento de personajes arquetípicos, como "el sabanero", el cual es representativo de una sociedad que se mira así misma como un colectivo producto $y$ poseedor de lo más distintivo de la cultura guanacasteca. Además de ello hay otros bienes o productos culturales como la música, las bombas, las retahílas, las festividades patronales (donde el Cristo de Esquipulas juega un papel central), la gastronomía "típica" (con una serie de platillos que son considerados por la población del cantón como autóctonos, aunque se enmarcan en una tradición gastronómica mesoamericana en primer momento, y mestiza luego, donde el maíz es un elemento fundamental en la dieta). Por estas $y$ otras características particulares de la cultura santacruceña, se le ha concedido al cantón el título de Ciudad Folklórica de Costa Rica, acreditado por el expresidente de la República Daniel Oduber en el año de 1974, ya que es un cantón en el que la cultura tradicional guanacasteca encuentra una variante de gran expresividad $y$ riqueza cultural.

No obstante, hay también, desde fines de la década de 1980, paralelamente, un desarrollo de la actividad turística de características extensivas e intensivas, ubicando al cantón en las posiciones más altas en índices de desarrollo turístico, y por lo tanto desarrollo inmobiliario como actividad necesaria del tipo de turismo que se desarrolla en la zona. Este fenómeno económico ha provocado una reconversión productiva, dando un salto de actividades primarias, fundamentalmente agropecuarias, a actividades terciarias, donde el turismo es el eje vertebral de la nueva estructura económica. Por lo tanto, se han dado cambios en las dinámicas sociales y culturales relativamente significativos, de la mano de la llegada de turistas extranjeros, o bien del arribo de extranjeros que deciden residir en la región, atraídos sobre todo por la riqueza ecológica y natural.

En tal sentido, la cultura tradicional del cantón se ha modificado sustancialmente, $y$ además se ha convertido en un atractivo turístico, comercializándose $y$ vendiéndose como espectáculo o como souvenir, y por lo tanto vaciándose de significado, a su vez, se han generado reacciones de revitalización de la "cultura local" como mecanismo de defensa frente a la amenaza de la aculturación o la homogenización cultural que plantea el contacto directo con la cultura extranjera, principalmente la norteamericana.

Por tanto, hay una relación tensionada entre cultura local-popular y desarrollo turístico. Tal relación es el objeto de reflexión de este artículo. En un primer apartado describimos algunas de las principales características culturales de la población santacruceña, con tal de aproximarnos a una definición posible de sus particularidades culturales, las cuales le han hecho ser catalogada como "cuna del folklore guanacasteco" o Ciudad folklórica de Costa Rica. En un segundo apartado se reflexiona acerca del desarrollo turístico del cantón, y su posicionamiento como destino turístico de importancia. Por último, se reflexiona sobre la relación entre cultura local-popular y desarrollo turístico, en el marco del proceso de globalización, evaluando las características y consecuencias que la reconversión económica del cantón ha tenido sobre la esfera cultural. 


\section{LA CULTURA LOCAL EN SANTA CRUZ DE GUANACASTE: CIUDAD FOLKLÓRICA DE COSTA RICA}

Santa Cruz es el tercer cantón de la provincia guanacasteca, ubicada en el pacífico norte de Costa Rica. Sobre esta región recayó la influencia cultural mesoamericana durante la época precolombina. Tal influencia se pone de manifiesto tanto en los vestigios arqueológicos como en algunas prácticas culturales que aun perviven en su población, relacionadas con algunas comidas tradicionales (en su mayoría basadas en el maíz), tradiciones orales, y en mucho con el léxico, el cual se encuentra ampliamente permeado por la raíz lingüística náhuatl (Quesada, 1991).

Posteriormente a la época precolombina, con el advenimiento de los conquistadores europeos, se inicia e intensifica el proceso de configuración de la sociedad guanacasteca en general, y santacruceña en particular. Este proceso estuvo signado por la estructura sociopolítica, y socioeconómica que generan los colonos españoles, en su mezcla e interacción con los pobladores autóctonos indígenas, y los esclavos negros traídos de África y de las Antillas. En un estudio interesante sobre la cultura y la historia tanto guanacasteca como santacruceña, Roberto Cabrera Padilla realiza el siguiente señalamiento sobre el devenir histórico de Guanacaste:

Los estudios e investigaciones en ciencias sociales sobre Guanacaste hablan de la Gran Nicoya o sector de influencia mesoamericano en el periodo precolombino; de conquista en el periodo colonial... de haciendas $y$ latifundios... de las estructuras de poder $y$ de la hegemonía en la producción agropecuaria... de folclore $y$ tradiciones populares ligados a la cultura sabanera y el mestizaje europeo, indígena, $y$ negroide (Cabrera, 1989: 13).

Estas particularidades histórico-sociales son, en buena medida, las que han dado paso a la configuración de la cultura popular santacruceña actual. Sin embargo, es preciso caracterizar tal cultura popular. Por tanto ¿Cuáles son esas particularidades que definen la cultura popular santacruceña en el marco históricosocial contemporáneo?

\section{PARTICULARIDADES DE LA CULTURA POPULAR SANTACRUCEÑA}

La problemática sobre la definición de la cultura popular, y sobre cuán válido resulta el concepto de cultura popular en tiempos de globalización, brinda elementos para pensar la cultura popular santacruceña. En principio lo popular fue definido en contraposición a la alta cultura moderna, generalmente vinculada al desarrollo de las ciudades. En este sentido, lo popular resulta de la construcción cultural que elaboran las sociedades alejadas de la metrópolis, cuyos referentes materiales son su entorno inmediato, el cual está caracterizado por la ruralidad, y por índices bajos de desarrollo. Sobre esta problemática definición Jean Franco plantea lo siguiente:

Antes lo popular fue un indicador de la diferencia latinoamericana, una diferencia que según la clase más cercana a la metrópolis se determinaba por la distancia de la metrópolis y que se percibía como el fundamento de la categoría de nación, ya fuera el gaucho independiente o la población rural auténtica. Pero la cultura popular servía igualmente como indicador de subdesarrollo; era pre-ilustración, prealfabetismo, era tradición como lo opuesto a progreso, atraso como lo opuesto a modernidad... (Franco, 2008: 2).

En principio, se podría plantear que la cultura santacruceña inicia situándose claramente en el sustrato de lo que se entendía como popular. Una cultura casi independiente del resto de Costa Rica, con un desarrollo muy particular. En esta cultura destaca la figura del "Sabanero", como personaje "auténtico" del pueblo, homologable al gaucho argentino o al charro mexicano, el cual es todo un arquetipo de la identidad cultural guanacasteca en general y santacruceña particularmente. Sobre esta figura emblema Soili Buska señala que: 
The guanacastecan also was to be a macho, usually sabanero, or peón, but definitely a hard-working man. The sabanero was becoming the symbol of the province, and would later become an object of the scholarly studies. Marc Edelman has suggested the sabanero cult was originally created to boost pride in hacienda work, because that was the only effective way of holding workers on the ranches before the economic crisis of the mid 1930s... "Sabanero pride" did not exist in a similar sense in Nicaragua (Buska, 2006:2009)1.

Este producto cultural, que se ha desarrollado en el cantón de Santa Cruz está en estricta relación con una estructura económica donde la ganadería, y sus actividades paralelas, así como la agricultura basada en buena medida en el maíz, marcan el derrotero, o la base material que ha tomado la cultura de esta población desde el siglo XIX, y gran parte del XX, $y$ que ha servido como fuente para la creación de múltiples productos culturales, que abarcan buena parte de todas las esferas de la vida de esta población (Quesada, 1991) ${ }^{2}$.

Por tanto, durante buena parte de la historia del cantón santacruceño, sus particula-

El guanacasteco también ha sido un macho, por lo general sabanero o peón, pero definitivamente un hombre trabajador. El Sabanero se ha convertido en el símbolo de la provincia, y más tarde se convertiría en un objeto de los estudios académicos. Marc Edelman ha sugerido que el culto sabanero fue originalmente creado para impulsar el orgullo en el trabajo de hacienda, porque era la única forma eficaz de explotación de los trabajadores en las fincas antes de la crisis económica de mediados de 1930 ... "Orgullo Sabanero" no existía en un sentido similar en Nicaragua (Traducción propia).

2 En la obra El español de Guanacaste de Miguel Quesada Pacheco, se hace un recuento de una gran cantidad de palabras usadas en la provincia en general y en Santa Cruz en particular, de las cuales un buen numero están referidas a cuestiones o elementos relacionados con la práctica de la ganadería y de la agricultura. El autor destaca además la influencia indígena, española y negra en las estructuras lingüísticas y lexicografitas de la provincia. ridades culturales han seguido el patrón que señala Jean Franco en torno a lo que se ha considerado tradicionalmente como popular. Además de ello, las producciones culturales que se consideran representativas del cantón, y que diversos agentes sociales, como folkloristas, educadores, entre otros, idealizan y aíslan del resto del país e incluso de la provincia, con tal de autoreferenciar la identidad santacruceña, como una identidad local diferenciada, propia y auténtica. Por tanto, la cultura del cantón, se comporta en consonancia con lo que podríamos considerar propio de una identidad local, la cual en palabras de Patricia Safa se podría definir de la siguiente manera:

... si bien las identidades locales se basan en delimitaciones geopolíticas históricas conformadas - delegaciones, barrios, pueblos, colonias - son sobre todo el resultado de un proceso de construcción social cultural... Las identidades locales son construcciones imaginarias, una invención, en donde no interesa necesariamente la correspondencia con los elementos objetivos o la verdad histórica para su legitimación o eficacia (Safa, 1998: 173).

En Santa Cruz, tales representaciones de la cultura popular que forman parte de identidad local son de vieja data. Se remontan al pasado colonial y a los inicios de la formación del Estado-Nación costarricense. Más de cien años en los cuales esta cultura se ha forjado. Sin embargo, existe una suerte de naturalización de la cultura santacruceña:

Los trabajos de algunos folcloristas locales procesan algunos fragmentos que sobreviven - mutilados y contaminados - de ese caudal cultural acumulado. Fragmentos que algunos idealizan $y$ vuelven intemporales para justificar una identidad que les es negada o que se intenta conservar como patrimonio nacional por otros (Cabrera, 1989: 81).

Este fenómeno, señalado por Cabrera se desarrolla en un marco de globalización en el 
que los patrones culturales $y$ valorativos han cambiado lo suficiente como para visualizar que la cultura tradicional-popular no es estática, sino dinámica y cambiante. A pesar de ello, muchas de las tradiciones santacruceñas han sido rigurosamente documentadas, $y$ los folcloristas, entre otros agentes sociales, intentan rescatarlas e inculcarlas a las generaciones más jóvenes.

Entre las principales características de la cultura popular del cantón, que sirven como base de la construcción de la identidad cultural de los santacruceños, destacan una serie de producciones culturales particulares, que forman parte de un conglomerado simbólico y material idealizado, cuya función es una reafirmación y auto-representación de este colectivo social.

Hay varias obras que realizan un recuento de las principales creaciones o productos culturales que se consideran propios del cantón de Santa Cruz. Una de ellas es Santa Cruz: el paraje de Diriá, de un autor santacruceño, Dr. Edgar Leal Arrieta, obra que recoge una serie de tradiciones y prácticas culturales que se le atribuyen exclusivamente a los pobladores del cantón, a pesar de que se podría argumentar la relación de tales producciones culturales en una tradición más amplia característica de la región mesoamericana. Entre las principales tradiciones y costumbres que documenta Leal Arrieta destacan la devoción por el Santo Cristo de Esquipulas, el cual es un producto del sincretismo indígena-español, las "Fiestas de Enero", que se desarrollan en honor al Cristo de Esquipulas, y que fueron declaradas Fiestas Típicas Nacionales por el expresidente de la República Lic. Daniel Oduber en el año de 1974, al mismo tiempo que se declaró al cantón de Santa Cruz el título de Ciudad Folklórica de Costa Rica. Asimismo, Leal documenta las "lagarteadas en Ortega", actividad en la cual los hombres, $y$ algunas mujeres, ambos vecinos del pueblo de Ortega, perteneciente al distrito de Bolsón de Santa Cruz, acostumbran el domingo de Resurrección, cazar un par de lagartos $y$ exhibirlos a los visitantes. Otras tradiciones son las coyoleras, lugares en los que se vende la bebida conocida como Coyol, extraída de la palmera que recibe el mismo nombre (Leal, 1998).
Además de lo anterior, en el cantón de Santa Cruz han surgido una serie de folkloristas que buscan mantener vivas las tradiciones antes reseñadas, estos movimientos van desde la música hasta lo culinario, desde la danza hasta la poesía, así como las artesanías. Todos estos movimientos folcloristas constituyen manifestaciones que buscan rescatar distintas expresiones consideradas como propias de la cultura del cantón, la danza y otras expresiones artísticas que se pueden ubicar dentro de esa serie de elementos que forman la cultura local ${ }^{3}$.

En una obra reciente, titulada Me lo dijo el río, otro folklorista y comunicador, Hernán Gutiérrez Oviedo, realiza una valiosa documentación basada en tradición oral, acerca del modo de vida que se desarrolló en Santa Cruz entre 1930 y 1970. Mediante una serie de entrevistas e historias de vida, realizadas a personas mayores de setenta años (el menor de los informantes nació en 1936 y el mayor en 1908) Gutiérrez profundiza múltiples de las tradiciones del cantón de Santa Cruz. En esta obra se

Dentro de estos movimientos folcloristas destacan entre otros, el Taller de Danza Folclórica Náhuatl, el grupo de bailes típicos Flor de Caña, los grupos musicales como Los de la Bajura, el trío Nacascolo, el trío Contradanza, grupos de marimbas como la Marimba Orquesta Maribel, o bien los marimbistas Alejandro Briceño, Ulpiano Duarte, el grupo Los Pochos de Ortega, al igual que una serie de marimbistas anónimos, de cada pequeño poblado. En las letras han despuntado escritores como Edgar Leal Arrieta, o bien Inocente Gutiérrez, Rodolfo Solórzano Bonilla, Héctor Chavarría Carrillo, cada uno ha escrito una interesante obra que abarca desde la prosa hasta la poseía. Así mismo la comunidad de Guaitil es reconocida por su constante trabajo en la confección de artesanía basada en las tradiciones que datan desde tiempos precolombinos, $y$ por otro lado, en las comidas típicas destaca la oferta gastronómica liderada por la Cooperativa Las Tortilleras, una asociación de mujeres que ha visto en el rescate de las tradiciones culinarias una posibilidad de trabajo remunerado. Además, dentro de estos movimientos folcloristas, es necesario destacar que cada año, el 14 de enero, inicia la celebración de las fiestas populares del cantón, las cuales fueron declaradas Fiestas Cívicas Nacionales, por la riqueza cultural que poseen, $y$ por la importancia que poseen como espacio de manifestación de las más diversas expresiones de la cultura local del cantón. 
rescatan buena parte de la vida cotidiana del cantón, desde el trabajo, la alimentación, la vida en las haciendas ganaderas, la vestimenta, las festividades, las relaciones familiares $y$ de pareja, las costumbres religiosas, los valores de la época, entre otras (Gutiérrez, 2008).

En síntesis lo central de la cultura popular santacruceña está en la imbricación o mestizaje de lo indígena, lo negro y español, al igual que en buena parte de América Latina, $y$ en el papel central de la gran hacienda ganadera, la cual ha articulado la estructura productiva que ha servido como base material de la cultura popular. Estas características la diferencian en buena medida del resto de Costa Rica, pero la asemejan a varias poblaciones de lo que tradicionalmente se ha denominado la región mesoamericana. Sobre ello, Cabrera plantea lo siguiente:

La cultura guanacasteca $y$, santacruceña en particular, en su historia y comunicación social, llega a nuestros días a través de la dinámica socioeconómica de poblaciones surgidas en un espacio de encrucijada, de frontera o de paso. $\mathrm{Su}$ cultura es, por tanto, el conjunto de procesos, fenómenos y acciones que tienen una larga historia agraria y ganadera... partiendo del siglo XVII hasta los inicios del presente siglo, entran de nuevo en un proceso de oscilaciones que condicen a la integración de la producción agropecuaria guanacasteca y su mercado interno, con las necesidades socioeconómicas de la región centroamericana, fusionada al mercado internacional (Cabrera, 1989: 66-67).

De modo que la identidad cultural santacruceña, y lo que se podría catalogar como cultura popular, presenta - entre otras - las características antes citadas, estrictamente ligadas a la dinámica económica y productiva que tradicionalmente han sido los ejes de la estructura económico-social del cantón. Tal estructura ha experimentado en los últimos años un proceso de cambio sustancial, pasando de la preponderancia del sector agropecuario a la centralidad del sector servicios, donde el turismo juega un papel vertebral.

\section{RECONVERSIÓN PRODUCTIVA DEL CANTÓN DE SANTA CRUZ}

A finales de la década de 1980 inicia en el cantón de Santa Cruz un proceso de reconversión productiva, donde las actividades tradicionales agropecuarias empiezan a retroceder debido al cambio en el estilo nacional de desarrollo del país.

A partir del primer gobierno de Óscar Arias (1986-1990), los ejes centrales de la política de desarrollo del país pasan a ser la profundización de las exportaciones de productos no tradicionales, iniciada con Luis Alberto Monge (1982-1986), la tecnología de avanzada, la atracción de Inversión Extranjera Directa (IED) y el desarrollo del sector servicios en el que el turismo juega un papel vertebral. Este cambio en la estrategia de desarrollo del país se da en un marco de factores condicionantes externos importantes, entre los cuales cabe citar el inicio de los procesos de paz en la región centroamericana, el avance del pensamiento neoliberal en la política internacional, liderado por EEUU y los gobiernos afines, $y$ un proceso de globalización económica y cultural. Es preciso señalar algunos indicadores importantes que nos permiten aproximarnos a la dimensión del desarrollo turístico que ha experimentado el cantón de Santa Cruz. Para reafirmar lo dicho, observemos la siguiente tabla: 


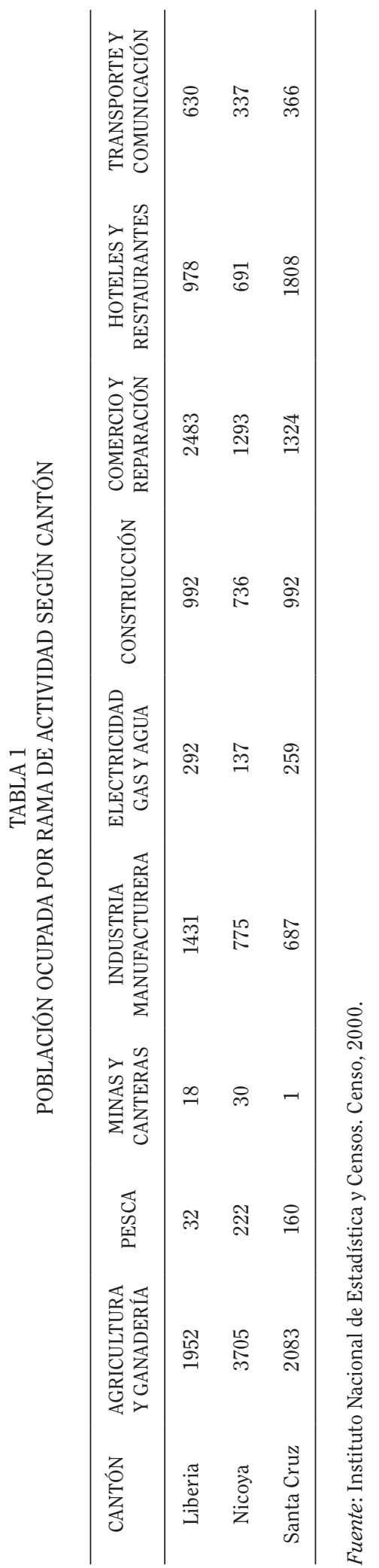


Según la tabla anterior, basada en datos del censo del 2000, para el cantón de Santa Cruz, la población ocupada en el sector servicios, plausiblemente relacionados con el sector turístico es casi igual a la ocupada en el sector agropecuario, lo cual es llamativo por dos razones. Primero, el sector servicios ha crecido y el sector agropecuario ha disminuido, y segundo, si se comparan los datos con los de otros cantones vecinos como el de Nicoya o Liberia, dejan ver que hay un cambio importante, sobre todo respecto a Nicoya, el cual se mantiene en una estructura productiva tradicional, presumiblemente similar a la de Santa Cruz antes del inicio de la llegada y profundización del turismo.

En primera instancia cabe señalar que la cantidad de población ocupada en el sector terciario, en el cual destacan los hoteles y restaurantes, así como el comercio al por mayor $y$ detalle, han crecido considerablemente en relación con la población ocupada en el sector agropecuario, lo cual lo podemos observar en el siguiente gráfico:

\section{GRÁFICO 1}

POBLACIÓN OCUPADA POR RAMA DE ACTIVIDAD PARA EL CANTÓN DE SANTA CRUZ
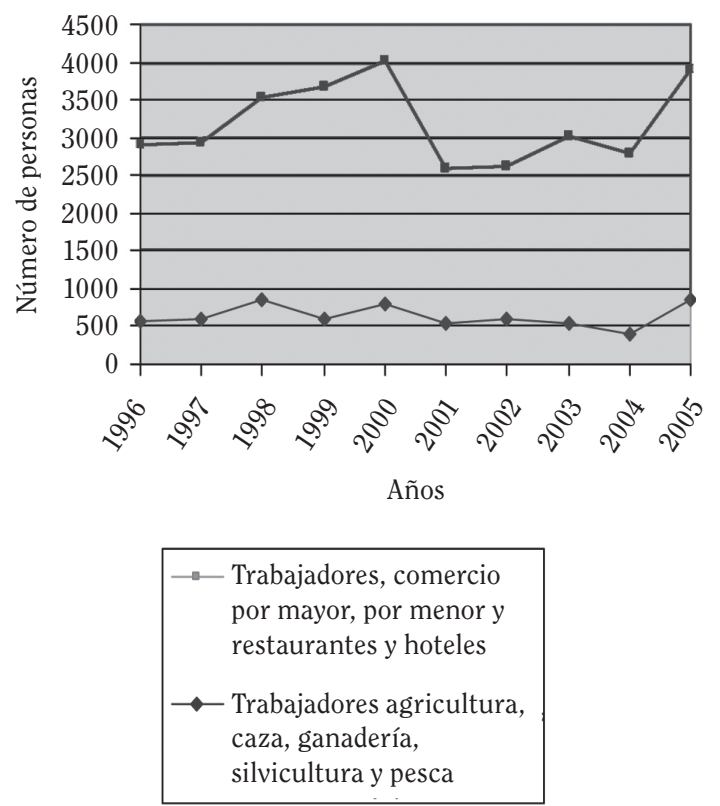

Fuente: Elaboración propia con base en datos del Portal de Datos Tendencias del Desarrollo Costarricense, Observatorio del Desarrollo de la Universidad de Costa Rica.
Como se observa en el gráfico 1, el número de personas ocupadas en actividades relacionadas con el sector turístico presenta una diferencia considerable cuando se le compara con las personas ocupadas en el sector agropecuario. Esto es un indicador importante del proceso de reconversión productiva que ha experimentado el cantón santacruceño en los últimos años.

Por otro lado, a la par del desarrollo turístico, se ha dado un desarrollo del sector de la construcción que también resulta considerable. En la actividad de la construcción, en el 2007, el cantón de Santa Cruz ocupa en el nivel nacional, el segundo lugar en metros cuadrados de construcción, según datos del Colegio Federado de Ingenieros y Arquitectos, tal como se aprecia en la siguiente tabla:

TABLA 2

DISTRIBUCIÓN DE FRECUENCIA ABSOLUTA Y RELATIVA DE $\mathrm{M}^{2}$, DE LOS CINCO CANTONES CON MAYOR ÍNDICE DE CONSTRUCCIÓN EN EL PAÍS ENERO-DICIEMBRE, 2007

\begin{tabular}{cccc}
\hline PROVINCIA & CANTÓN & FR. ABS. & FR. RELAT. \\
\hline Alajuela & Alajuela & 586819 & $6,93 \%$ \\
Guanacaste & Santa Cruz & 570695 & $6,74 \%$ \\
Puntarenas & Garabito & 565181 & $6,68 \%$ \\
Guanacaste & Carrillo & 481672 & $5,69 \%$ \\
Heredia & Heredia & 456134 & $5,39 \%$ \\
\hline
\end{tabular}

Fuente: Colegio Federado de Ingenieros y Arquitectos, 2007.

Como se observa en la tabla anterior, el crecimiento infraestructural del cantón es destacable a un nivel nacional, $y$ se encuentra relacionado con el proceso de reconversión productiva de Santa Cruz, en el tanto, la antigua hacienda ganadera va desapareciendo, afloran complejos habitacionales, comerciales, entre otros, potenciados por el desarrollo turístico de la región. Este cambio en la base productiva del cantón, la cual se encontraba en función del sector agropecuario, $y$ que se reorienta hacia el sector servicios, se manifiesta en la esfera cultural, tales cambios se analizan en el siguiente apartado. 


\section{CULTURA LOCAL Y TURISMO EN SANTA CRUZ: EL RESCATE DE LA CULTURA EN FUNCIÓN DEL TURISMO}

En el desarrollo histórico-social del cantón de Santa Cruz, la base material de la cultura local ha constituido, hasta finales de 1980, una estructura productiva basada en las actividades agropecuarias, donde la hacienda ganadera ha jugado un papel central, $y$ ha dado paso a una serie de producciones culturales que se han idealizado y casi des-historizado, (pues cuando se habla de cultura popular en Santa Cruz se hace referencia inmediatamente al personaje típico o arquetípico del sabanero, a las corridas de toros, a las comidas basadas en el maíz, bailes tradicionales, entre otras particularidades características de la tradición de la zona).

En este contexto cabe preguntarse cuál es el papel de la cultura local en un marco de reconversión de la estructura productiva, desarrollo turístico, y aun en un contexto más amplio de globalización. Es decir, si la base material de la cultura local-popular eran las actividades tradicionales agropecuarias, ¿qué ha pasado con esa cultura cuando esta base se ha modificado?

En un estudio de interés, titulado Percepciones de la población guanacasteca sobre la actividad turística en la región chorotega 2005, el cual se realizó entre 400 personas $^{4}$, los guanacastecos entrevistados expresan distintas opiniones sobre la actividad turística y su impacto en la cultura de la zona. Sobre la relación turismo y cultura local-popular los entrevistados en este estudio señalan que:

... 52\% de los entrevistados percibe un aumento en la pérdida de la identidad local (ser guanacasteco) y un $71 \%$ percibe a la vez un aumento en la imitación y copia de costumbre y hábitos extranjeros

4 Según este estudio, se seleccionaron 30 segmentos censales en forma aleatoria, con habitantes de las denominadas unidades de planeamiento turístico del Guanacaste Norte y Sur: Santa Cruz, Nicoya, Mansión, Comunidad, Sámara, Nosara, Filadelfia, Tamarindo, Playa Hermosa, Caimital, Sardinal, Carrillo, Guardia, Hojancha y Liberia. (comidas, ropas, gustos, estilos de vida). Paralelo a lo anterior los entrevistados consideran que el interés por impulsar programas para rescatar esta identidad guanacasteca, tanto los valores como las historias de las comunidades "apenas se mantiene igual" $y$ no han aumentado como es lo deseable y necesario para contrarrestar la erosión de la cultura e identidad local, que pueden ser afectadas por la actividad turística (González Calvo, 2005: 259).

Existe una percepción presumiblemente generalizada de que el turismo y la llegada de extranjeros, que arriban al cantón tanto de paso como de manera permanente, han tenido efectos nocivos sobre la cultura local. Esto es plausible, sin embargo, también hay que resaltar que existe una relación de la cultura local-popular no solo con los turistas extranjeros, sino que también se desarrolla en el marco del proceso de globalización, en el cual los referentes de construcción de la identidad no son necesariamente la realidad inmediata, sino que también, como plantea Giddens, la identidad se construye no ya en referencia a los anclajes de tiempo y de espacio tradicionales, sino que se toman con referencia de estilos de vida que trascienden el tiempo y el espacio (Giddens, 1997).

De lo anterior resulta que para pensar la relación entre desarrollo turístico y cultura popular-local, es necesario situarla no solo en el proceso de reconversión productiva, sino también, en el marco del proceso de globalización. Por tanto, una derivación de la consideración anterior, nos lleva a afirmar con Elsa Flores Ballesteros que:

... los procesos de globalización conmueven los cimientos de los sistemas estéticos, y más allá, los de las culturas estéticas mismas, así como las tramas de las identidades modernas de distinto tipo. Pero esta situación, a nivel planetario, no implica de (sic) la desaparición de aquellos, sino su reacomodación en función de un nuevo ordenamiento, cuya resolución aun no se divisa totalmente (1998: 145). 
Siguiendo el planteamiento de Flores Ballesteros, se observa precisamente en el cantón de Santa Cruz, un reacomodo de la cultura popular-local, a la nueva estructura productiva del cantón. Las tradiciones culturales populares se han resignificado, idealizado, y en última instancia se han comercializado para venderlas como un bien cultural sobre todo al turista extranjero. Este proceso de comercialización de la cultura popular se evidencia en los hoteles que ofrecen espectáculos donde se retrata aquella cultura que se considera tradicional, caracterizada por bailes y música "típica”, espectáculos taurinos o ecuestres, con el fin de entretener a los turistas, o bien se ha desarrollado un comercio importante de artesanías, donde destaca la cerámica tradicional de herencia indígena, sobre todo en el poblado santacruceño llamado Guaitil, el cual se ha caracterizado por el desarrollo de toda una industria y comercialización de artesanías de influencia chorotega, tanto en su técnica como en sus materiales, aunque no tanto en sus motivos. En otras palabras se ha generado en buena medida un turismo de corte cultural, aunque no sea la principal atracción de la zona.

Este fenómeno cumple dos funciones. Por un lado, crear un producto distintivo de la zona que se pueda comercializar en el marco del desarrollo turístico, y por otra parte, es un pretexto para rescatar la cultura popular-local que se considera como cultura tradicional, por lo que se han creado espacios donde los folcloristas han desarrollado un trabajo de registro de la cultura popular-local. Estos fenómenos se han desarrollado tanto en los poblados cercanos a la zona marítimo terrestre, zona donde se ha desarrollado en mayor medida el despliegue del sector turístico, y también han sido una válvula de escape en las zonas que al no estar cerca de las costas, $y$ al ver sus estructuras productivas agropecuarias casi totalmente desarticuladas, han tomado como una de las pocas formas productivas, la comercialización de la cultura tradicional, tal es el caso del poblado mencionado, Guaitil. También de la cabecera del cantón de Santa Cruz, el distrito primero que lleva el mismo nombre del cantón, donde un grupo de personas ha desarrollado un proyecto de rescate cultural, Al rescate cultural de Santa Cruz en función del turismo, el cual resulta de gran interés para observar la tensionada relación entre cultura local y turismo.

Este proyecto busca captar turistas para el distrito de Santa Cruz, en particular para el centro poblacional, pues la mayor parte de los turistas se dirigen a la zona marítima terrestre. Tal captación se pretende realizar mediante la creación de una oferta cultural como mercancía turística. En ese sentido, se trata del reacomodo de la cultura localpopular como mercancía turística. Sobre este tema, Allen Cordero plantea lo siguiente:

En efecto la cultura turística dominante se apropia de los espacios de expresión de la cultura turística local, trata de rearticular contenidos culturales turísticos de las comunidades asentadas en esos territorios, $y$ busca presentarlos de acuerdo con los gustos de los turistas internacionales (2006: 126).

En tal sentido, el proyecto Al rescate cultural de Santa Cruz resulta como un mecanismo para rearticular la cultura popular al proceso de reconversión productiva del cantón en el que el sector turístico juega un papel central.

En el proyecto en cuestión se parte de la consideración de ofrecer a la ciudad de Santa Cruz como un producto turístico. Esto se pretende lograr haciendo énfasis en la cultura popular o folklórica de la zona como una mercancía comercializable. Para ello se realizó todo un estudio de mercado, así como del perfil de los turistas y un inventario de los atractivos culturales de la ciudad, con el fin de poder configurar una oferta consolidada de bien cultural dirigido al turista, sobre todo extranjero.

De esta manera, el proyecto Al rescate cultural de Santa Cruz (ver anexo 1) caracteriza su producto de la siguiente manera:

El producto a desarrollar tendrá las siguientes características:

"SANTA CRUZ CIUDAD FOLKLÓRICA", deberá ser una secuencia de productos de entretenimiento y cultura, donde el turista a través del recorrido por la ciudad se convierte de espectador a participante de 
la historia e idiosincrasia de la ciudad de Santa Cruz y sitios aledaños ${ }^{5}$.

En la cita anterior se puntualizan los objetivos del proyecto, evidencia muy bien, la parte de la relación entre la cultura popularlocal santacruceña, idealizada, y el desarrollo del turismo en un marco de globalización. Hay un énfasis fuerte en crear un "producto cultural" que sea divertido, mágico, enérgico, ocurrente, propio de lo que de forma estereotipada se ha considerado la cultura popular latinoamericana en general.

Por tanto, la relación entre cultura y turismo en la ciudad de Santa Cruz, está claramente en función de la comercialización de la cultura popular, con el fin de lograr una oferta turística diferenciada.

Dentro de este fenómeno, es posible observar, que existe en la oferta turística de muchos hoteles, orientados sobre todo a los turistas extranjeros, una variedad de bienes culturales comercializables, que tienen como principal objetivo ampliar la gama de servicios con la cual podría contar la oferta del hotel, $y$ crear un "producto turístico distintivo basado en ciertas particularidades culturales".

Un ejemplo concreto de ello es el hotel Hacienda Pinilla, en la comunidad de Pinilla, Santa Cruz, el cual es un ambicioso proyecto fundamentalmente orientado a los turistas extranjeros, y cuenta con un área de 4500 acres (1821,08 hectáreas); dentro de sus instalaciones hay condominios, hoteles, restaurantes, canchas de golf, lagos artificiales, $y$ villas cuyo precio de alquiler va desde 345 a 1155 dólares por persona por noche, todo ello frente a la costa del pacífico santacruceño.

En este hotel, dentro de los servicios que ofrece, se encuentra el evento denominado Fiesta Sabanera, el cual es un espectáculo basado en diversas tradiciones de la zona, como música típica, cimarrona, payasos, corridas

El estudio titulado Al rescate cultural de Santa Cruz fue elaborado por la entidad llamada Internacional Tourism Management Costa Rica, agosto, 2004. de toros, carreras de caballos, $y$ danzas tradicionales, cuyo principal objetivo es el entretenimiento de los turistas ${ }^{6}$. Este es un ejemplo interesante sobre como la cultura tradicional se ha vaciado de significado, $y$ se ha convertido, en algunos casos en una especie de souvenir cultural, que se ofrece dentro de los paquetes turísticos.

En este sentido presumiblemente se reorientará el devenir de la cultura tradicional guanacasteca en general y santacruceña particularmente, acabada ya la base material de la cultura tradicional, ganadera y agraria, $y$ en el marco de la profundización del desarrollo turístico, $y$ del proceso globalizador. Una forma de vida se convierte en espectáculo: Santa Cruz como "parque temático" de su propio pasado.

\section{CONCLUSIONES}

Lo que se ha considerado como la cultura popular, local o tradicional del cantón santacruceño ha tenido su base material primeramente en el proceso de choque y mestizaje de los grupos indígenas autóctonos, los conquistadores europeos y los negros llegados de África y de las Antillas, proceso de hibridación cultural que es la base de la cultura actual. Además de ello, la base material ha estado en la estructura productiva agropecuaria, donde la hacienda ganadera ha jugado un papel central en mucho de las producciones culturales de la región.

Los cambios que se han desarrollado recientemente ponen en entredicho esta cultura tradicional. El desarrollo turístico y el proceso globalizador han generado no solo la pérdida de aquella cultura popular centenaria, sino además la adopción de nuevos valores y patrones culturales. Por otra parte, hay ya procesos de hibridación donde de la misma manera, los extranjeros que se sitúan en zonas rurales, generalmente costeras y turísticas de nuestro país, toman patrones propios de las culturas tradicionales, generando una dinámica de transferencia recíproca.

http://www.haciendapinilla.com/fiesta_sabanera. html (con acceso al 24 de noviembre de 2009). 
Es necesario sugerir, que en este contexto, que la cultura local ha cambiado debido a los nuevos procesos socio-históricos y socio-económicos que ha experimentado esta región, fundamentalmente influidos por las transformaciones dadas en el marco del desarrollo turístico. Desde nuestra perspectiva, la cultura local del cantón ha tomado dos vertientes o direcciones principales: una primera de resignificación y revaloración, pues se llega a considerar como un bien intangible que es susceptible de ser comercializado, $y$ de obtener ganancias a partir de este producto cultural, esto ha motivado un impulso de las tradiciones locales puesto que funcionan como mercancía $y$ generan utilidades, lo cual deriva en la existencia de profesionales de la cultura local, o lo que es lo mismo folcloristas, que venden las manifestaciones culturales, $y$ las tradiciones en el mercado turístico.

La segunda tendencia, es la concepción de la cultura local como un bien que se encuentra en procesos de transformación, y de cambio, orientándose sobre todo a la desaparición, es por ello que muchos hablan de rescate de las tradiciones, o de la conservación de la cultura, dentro de esta tendencia destacan igualmente algunos folcloristas, e instituciones (escuelas, universidades, entre otras) que buscan fomentar la cultura local frente a la idea o la amenaza de una posible extinción de tal cultura.

Por tanto el papel que ha jugado la cultura popular santacruceña, en el marco de esta propia sociedad ha cambiado. Ya no son formas de hacer, actuar o pensar cotidianas, pues la base material de referencia ha cambiado. Ahora su función es más la de evocar a una época lejana, $y$ sin retorno, $y$ la de formar parte de una oferta de bienes culturales en función del turismo. Es plausible pensar que no mucho cambiará en el devenir de esta cultura local, pues este derrotero tiende a profundizarse, aunque aún no podemos plantear cual será el desenlace de esta tensión entre cultura popular-local y turismo globalizado. 


\section{ANEXO 1}

Los objetivos del proyecto Al rescate cultural de Santa Cruz, son los siguientes:

Los productos deberán mostrar la energía, la alegría, lo ocurrente y vivacidad del pueblo, estar disponibles durante todo el año, $y$ estar preparados para brindar un excelente servicio de calidad, no importando las condiciones climáticas de la zona.

Los productos deberán ser mágicos y envolver al participante, sin importar su idioma nativo.

Los productos deben mostrar no solamente la historia santacruceña, a través de sus manifestaciones culturales en la música, vestuario e idiosincrasia del "Santacruceño", sino también a través de la variabilidad de su gastronomía, en montajes autóctonos y ofreciendo variedad de platillos y bebidas vistosas, bien elaboradas y presentadas. También mostrar presentaciones artísticas con mucho colorido $y$ creatividad $y$ ofrecer una diversidad de opciones para actividades de un día.

El producto deberá ofrecer un precio acorde a la calidad del servicio y segmento de mercado, resaltando la tradicional hospitalidad y cocina santacruceña de tierra adentro $y$ de las zonas costeras en un ambiente tradicional y natural. Con personal capacitado $y$ dispuesto a ofrecer el mejor servicio y calidad al turista.

$\diamond$ Diversidad de actividades, en un marco de planta física tradicional y bien cuidada, limpia, con amplias zonas verdes, alamedas de grandes y tradicionales árboles, con espacios suficientes para parqueos, zonas de artesanía, áreas para pequeños pero acogedores restaurantes, en donde se especialice la comida criollas de tierra adentro y zonas costeras. Los restaurantes con sitios diseñados para espectáculos diarios de marimbas y danzas tradicionales.

$\diamond$ Ofrecer visitas a las haciendas donde los turistas puedan disfrutar de las actividades tradiciones como las montas de toro $y$ rodeos a la usanza Santa Cruceña y aprovechar el entorno natural para realizar caminatas, cabalgatas, observación de flora y fauna, observación de aves en la Parque Nacional Diriá.

$\diamond$ Planta (infraestructura) física, en donde conviven la arquitectura tradicional y la moderna en forma armoniosa.

$\diamond$ Deberá ser una nueva alternativa para el visitante en el aspecto cultural, que conlleva diversión y aprendizaje, con calidad de servicio competitiva a nivel internacional. 


\section{BIBLIOGRAFÍA}

Busca, Soili Iris. "Marimba por ti me muero". Region and nation in Costa Rica, 18241939. Department of History, Indiana University, 2006.

Cabrera Padilla, Roberto. Santa Cruz, Guanacaste. Una aproximación a la historia y la cultura populares. San José. Ediciones Guayacán, 1989: 274p.

Cordero Ulate, Allen. Nuevos ejes de acumulación y naturaleza: el caso del turismo. Buenos Aires. Consejo Latinoamericano de Ciencias Sociales, CLACSO, 2006.

Flores Ballesteros, Elsa. "Arte, identidad y globalización”. En: Bayardo R.; Lacarrieu M. Globalización e identidad cultural. Buenos Aires: Ediciones Ciccus, 1998.

Franco, Jean. La globalización y la crisis de lo popular. 2008. En: <http:// www.periodismo.uchile.cl/talleres/ teoríacomunicacion/archivos/ globalizacion.html> [con acceso al 6 de octubre de 2008].
González Calvo, Hugo. "Percepciones de la población guanacasteca sobre la actividad turística en la región chorotega 2005". En: González Calvo, Hugo. El turismo alternativo en la región centroamericana. Seminario Regional, Universidad Nacional, Guanacaste, Costa Rica, 2005.

Gutiérrez Oviedo, Hernán. Me lo dijo el río. Relatos breves sobre la historia de las comunidades vecinas al polo turístico Golfo de Papagayo 1930-1970. San José. Editorial UCR, 2008: 222p.

Internacional Tourism Management Costa Rica. Al rescate cultural de Santa Cruz. Agosto, 2004.

Leal Arrieta, Edgar. Santa Cruz: el paraje de Diriá. San José, Litho. 1998: 217p.

Quesada Pacheco, Miguel. El español de Guanacaste. San José. Editorial de la Universidad de Costa Rica, 1991: 235p.

Safa, Patricia. "De las historias locales al estudio de la diversidad en las grandes ciudades: una propuesta metodológica". En: Bayardo R.; Lacarrieu M. Globalización $e$ identidad cultural. Buenos Aires: Ediciones Ciccus, 1998. 